\title{
Perfil bacteriano de amostras microbiológicas de pacientes internados na Clínica Cirúrgica de um Hospital Universitário de Pernambuco
}

\author{
Ive Dias Mangueira Bastos ${ }^{\mathrm{a}}$, Bruna Dias Mangueira Bastos ${ }^{\mathrm{a}}$, Carine Freitas e Silva ${ }^{\mathrm{b}}$, \\ Katia Suely Batista Silva ${ }^{c}$, Carine Rosa Naue ${ }^{c^{*}}$
}

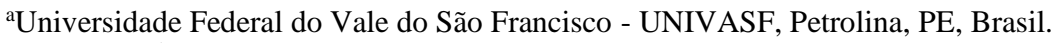

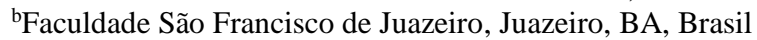

cHospital Universitário da Universidade Federal do Vale do São Francisco UNIVASF/EBSERH, Petrolina, PE, Brasil

Histórico do Artigo

Recebido em:

05/03/2020

Aceito em:

$16 / 04 / 2020$

Palavras-chave:

Perfil;

antimicrobianos;

bactérias; enfermaria

Keywords:

Profile;

antimicrobials;

bacteria; nursery

\section{RESUMO}

No Brasil, apesar de não haver uma sistematização dos dados, estima-se que, aproximadamente, 5 a $15 \%$ dos pacientes hospitalizados adquiram algum tipo de Infecção Relacionada à Assistência à Saúde (IRAS), sendo ela, em geral, a quarta causa de mortalidade. As IRAS apresentam uma enorme relevância para saúde pública, pois fatores como indivíduos imunocomprometidos associado ao surgimento da resistência a antimicrobianos, estão cada vez mais frequente no ambiente hospitalar, facilitando este tipo de infecção. O objetivo deste trabalho foi verificar a ocorrência e o perfil bacteriano de pacientes internados na Clínica Cirúrgica de um Hospital Universitário. $\mathrm{O}$ estudo foi realizado através da análise retrospectiva de exames de aspirados traqueais, hemoculturas e uroculturas de pacientes internados na Clínica Cirúrgica, no período de janeiro a dezembro de 2018. Os dados foram coletados por meio de impressos laboratoriais do próprio serviço e tabulados na planilha do Excel®, sendo divididos em amostras positivas e negativas e realizada análise descritiva com valores absolutos e percentuais. Em geral, as bactérias mais freqüentes, nas diferentes amostras, foram Pseudomonas aeruginosa, Escherichia coli, Staphylococcus coagulase negativa, Acinetobacter baumannii e Klebsiella pneumoniae. As espécies bacterianas com maior resistência foram Acinetobacter baumannii, Staphylococcus coagulase negativa, Klebsiella pneumoniae e Klebsiella oxytoca. Os dados deste trabalho permitem o conhecimento do perfil bacteriano encontrado nos pacientes internados, o que poderá nortear o tratamento das infecções e conseqüentemente auxiliar na diminuição da seleção de bactérias multirresistentes, auxiliando na prevenção e no controle das infecções hospitalares.

\section{Bacterial profile of microbiological samples from inpatients} Surgical Clinic of a University Hospital in Pernambuco

\section{ABSTRACT}

In Brazil, although there is no systematization of the data, it is estimated that approximately 5 to $15 \%$ of hospitalized patients acquire some type of infection related to healthcare, which, in general, is the fourth cause of mortality. HAIs are of enormous relevance to public health, since factors such as increasingly immunocompromised individuals, associated with the emergence of resistance to antimicrobials, are increasingly frequent in the hospital environment, facilitating this type of infection. The objective of this study was to verify the occurrence and the bacterial profile of patients admitted to the surgical clinic at the University Hospital. The study was carried out through the retrospective analysis of tracheal secretions, blood cultures and urine cultures of patients admitted to the Surgical Clinic, from January to December 2018. Data were collected through laboratory prints from the service itself and tabulated in the Excel® spreadsheet, being divided into positive and negative samples, and a descriptive analysis was performed with absolute and percentage values. In general, the bacteria that most occurred in the different samples were Pseudomonas aeruginosa, Escherichia coli, Coagulase negative Staphylococcus, Acinetobacter baumannii and Klebsiella pneumoniae. The bacterial species with the highest resistance were Acinetobacter baumannii, Coagulase negative Staphylococcus, Klebsiella pneumoniae and Klebsiella oxytoca. The data in this study allow the knowledge of the bacterial profile found in hospitalized patients, which may guide the treatment of infections and consequently reduce the selection of multidrug-resistant bacteria, helping to prevent and control hospital infections.

\section{Introdução}

\footnotetext{
* Autor correspondente: crnaue@yahoo.com.br (Naue C.R.)
} 
É conhecido que desde a década de 1990 o termo "infecções hospitalares" foi alterado para "infecções relacionadas à assistência em saúde" (IRAS) sendo essa nomeação um alongamento conceitual que incorpora as infecções adquiridas e aquelas relacionadas à assistência em qualquer ambiente (1). Essa nova terminologia abrange além das Instituições Hospitalares, os atendimentos ambulatoriais na modalidade de hospital ou domiciliar, que possam estar associados a alguns procedimentos assistenciais, seja ele terapêutico ou diagnóstico (2).

No Brasil, apesar de não haver uma sistematização dos dados, estima-se que, aproximadamente, 5 a 15\% dos pacientes hospitalizados adquirem algum tipo de infecção relacionada à assistência à saúde, sendo ela, em geral, a quarta causa de mortalidade (3). Uma pesquisa realizada em um Hospital Universitário verificou que a "taxa de prevalência de infecção hospitalar (IH) anual média é de 8,2\%, sendo 149 (29,1\%) pneumonias, $136(26,6 \%)$ infecções de corrente sanguínea, 87 (17\%) infecções do trato urinário, $57(11,1 \%)$ infecções de cateter central e 47 (9,2\%) infecções de sítio cirúrgico" (4).

Dentre os fatores que levam a IRAS, pode-se citar ouso de procedimentos invasivos, tratamentos com drogas que diminuem a imunidade do paciente, bem como a idade, principalmente os extremos, recém-nascidos e idosos. Aspectos como, obesidade, desnutrição, diabetes, também devem ser levados em consideração (5). Os tipos mais comuns de IRAS são as pneumonias associadas a ventilação, infecções do trato urinário associadas a cateter, infecções da corrente sanguínea associada a acesso central, infecções de feridas cirúrgicas e do trato gastrintestinal (2).

Essas infecções apresentam uma enorme relevância para saúde pública, pois fatores como, indivíduos cada vez mais imunocomprometidos, associado ao surgimento da resistência a antimicrobianos, estão cada vez mais frequente no ambiente hospitalar, facilitando o desenvolvimento destas patologias (6).

Vale ressaltar que a resistência microbiana vem sendo considerada uns dos maiores desafios aos sistemas de saúde contemporâneos, acredita-se que, mundialmente, cerca de 700 mil mortes sejam causadas anualmente pela resistência aos antimicrobianos (7).

Entre as bactérias resistentes causadoras de infecção em hospitais, tanto em países desenvolvidos como naqueles em desenvolvimento, destacam-se como as mais frequentes: Staphylococcus aureus resistentes à meticilina (MRSA), Enterococcus resistentes à vancomicina (VRE) e Staphylococcus coagulase-negativa (SCN) com perfil de sensibilidade reduzida e resistência à oxacilina e a cefalotina, podendo apresentar resistência cruzada a $\beta$-lactâmicos e outras classes de antibióticos (8-10).

Entre os bacilos Gram negativos (BGN), ganham destaque: Escherichia coli e Klebsiella pneumoniae produtoras de B-lactamase de espectro estendido (ESBL) incluindo a produção de carbapenemase, Escherichia coli resistentes a fluoroquinolonas, Pseudomonas aeruginosa e Acinetobacter baumannii resistentes aos carbapenêmicos e Enterobacter sp. resistentes a aminoglicosídeos, cefalosporinas de $3^{\text {a }}$ geração e fluoroquinolonas $(11,12)$.

Pesquisadores afirmam que, se não houver uma mudança de abordagem para conter o problema, até 2050, a resistência microbiana poderá causar mais mortes que o câncer. Diante desse pressuposto, uma alternativa para esta situação, seria traçar o perfil microbiano dos setores das Instituições de Saúde, dando norte para prevenção e tratamento de IRAS (13).

Sendo assim, o objetivo deste trabalho foi verificar a ocorrência e o perfil bacteriano em hemoculturas, secreções traqueais e uroculturas de pacientes internados na Clínica Cirúrgica de um Hospital Universitário. 


\section{Materiais e métodos}

O trabalho foi realizado em um Hospital Universitário no município de Petrolina-PE, que conta com o perfil assistencial de hospital geral de média e alta complexidade à comunidade adulta, com dimensionamento dos serviços assistenciais e de ensino e pesquisa.

Trata-se de um estudo retrospectivo, descritivo e documental com abordagem quantitativa, tendo como fonte de informação os exames de aspirados traqueais, hemoculturas automatizadas e uroculturas de pacientes internados na Clínica Cirúrgica, no período de janeiro a dezembro de 2018 , disponibilizadas pelo laboratório.

Os dados dos exames foram tabulados na planilha do Excel®, sendo divididos em amostras positivas e negativas e realizada análise descritiva com valores absolutos e em percentuais. Foram consideradas amostras positivas para urocultura quando a contagem de colônias foi maior ou igual a $100.000 \mathrm{UFC} / \mathrm{mL}$, para hemocultura quando houve qualquer crescimento bacteriano em um dos quatro frascos coletados por paciente e para cultura de aspirado traqueal quando a contagem de colônias foi maior ou igual a $10^{6}$ $\mathrm{UFC} / \mathrm{mL}$.

Nas amostras positivas foram analisadas as variáveis ocorrência de bactérias e o perfil de sensibilidade e resistência aos antibióticos testados. Os dados foram organizados e apresentados por meio de gráficos e tabelas.

As identificações das bactérias e os antibiogramas foram executados através do sistema automatizado PHOENIX, da BD, utilizando-se os painéis adequados. De acordo com a metodologia do Clinical and Laboratory Standards Institute (2018), os resultados foram classificados em sensível (S) e resistente (R).

O trabalho foi submetido ao Comitê de Ética da Universidade Federal do Vale do São Francisco (UNIVASF), sendo aprovado através do CAAE: 66493917.0.0000.5196.

\section{Resultados}

Foram realizados um total de 43 aspirados traqueais, sendo 12 negativos e 31 positivos. Em relação as hemoculturas foram realizadas 246, sendo 27 positivas e 219 negativas e para uroculturas foram realizadas 209, sendo 145 negativas 64 positivas.

Pode-se observar no (Figura 1), que as espécies de maior ocorrência em aspirados traqueais foram, Pseudomonas aeruginosa (29,3\%), Acinetobacter baumannii $(25,8 \%)$, Klebsiella pneumoniae $(16,1 \%)$ e Proteus mirabilis $(6,4 \%)$. Os restantes das espécies isoladas apresentaram uma ocorrência de 3,2\%. 


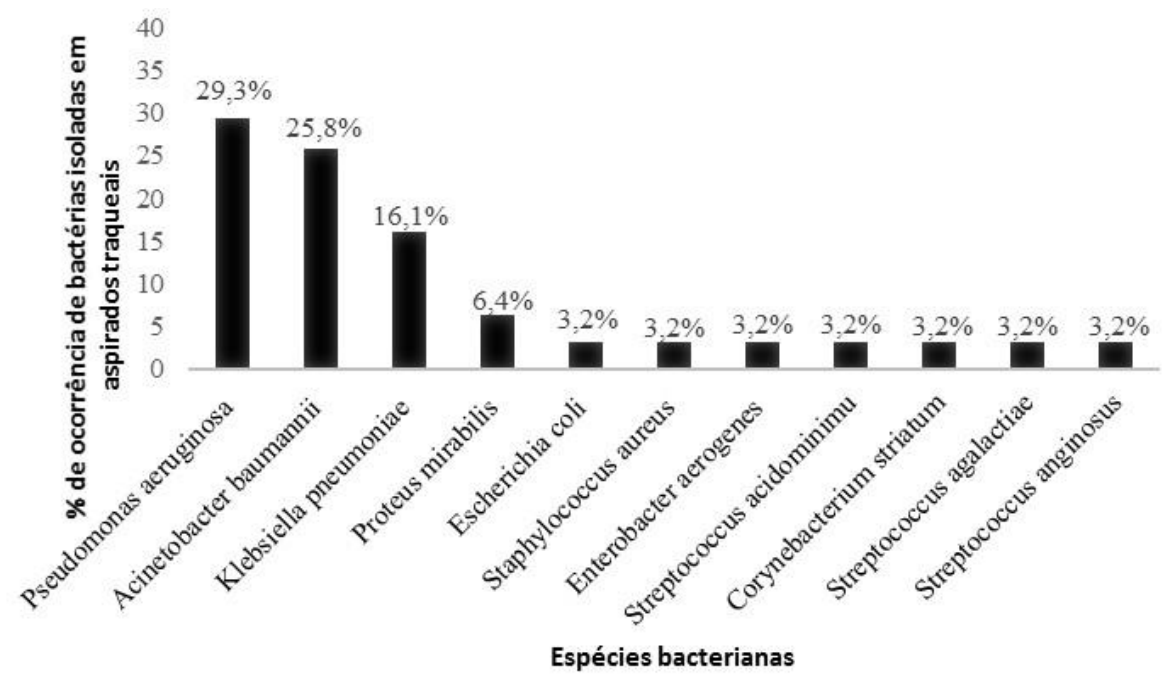

Figura 1 - Ocorrência de bactérias isoladas em aspirados traqueais coletados em pacientes internados na Clínica Cirúrgica de um Hospital Universitário, no período de janeiro a dezembro de 2018.

Para hemoculturas (Figura 2) as espécies mais frequentes foram Staphylococcus coagulase negativa (SCN) $(25,9 \%)$, Klebsiella pneumoniae $(22,2 \%)$ Candida glabrata $(11,1 \%)$, Enterococcus faecalis $(7,4 \%)$ e Staphylococcus aureus $(7,4 \%)$.

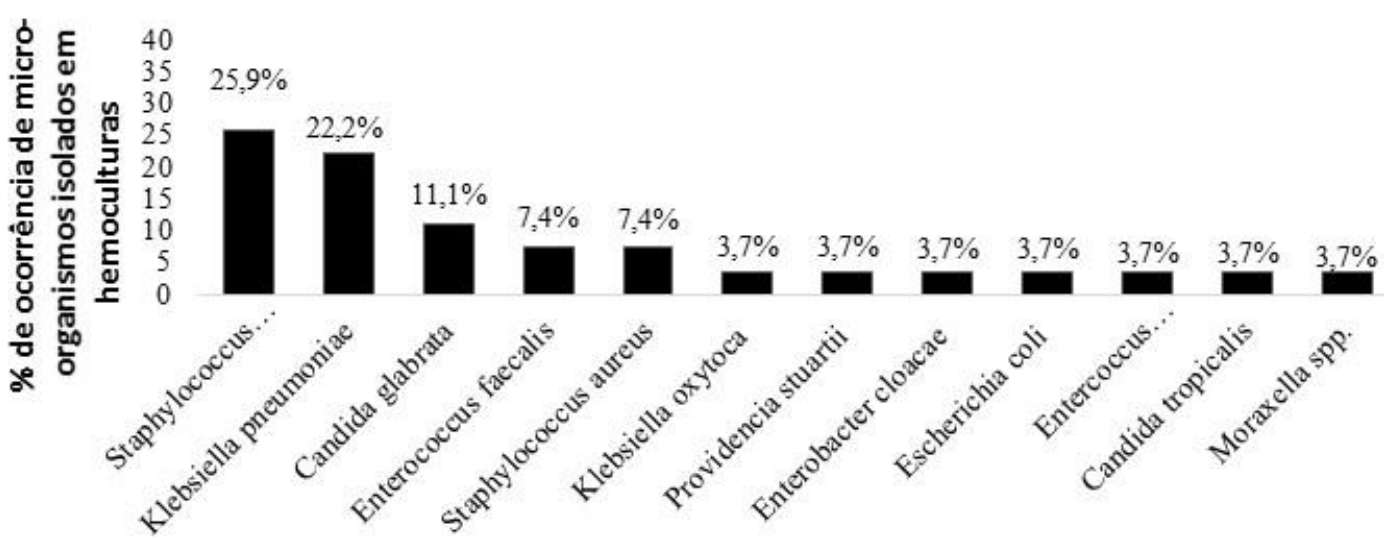

\section{Micro-organismos isolados}

Figura 2 - Ocorrência de micro-organismos isolados em hemoculturas coletadas em pacientes internados na Clínica Cirúrgica de um Hospital Universitário, no período de janeiro a dezembro de 2018.

Para uroculturas, como pode ser observado na (Figura 3) as espécies com maior frequência foram Escherichia coli (28,1\%), Klebsiella pneumoniae (25\%), Pseudomonas aeruginosa (12,5\%), Candida glabrata (6,2\%), Candida tropicalis (4,6\%). 


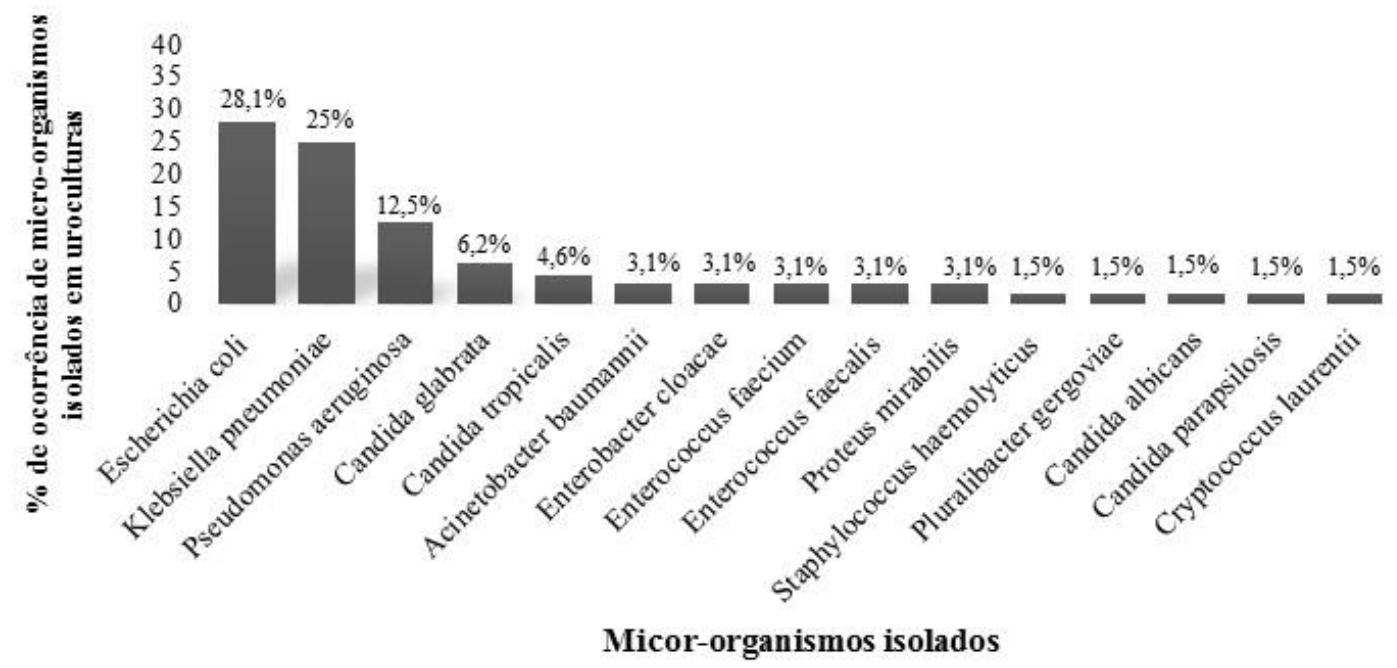

Figura 3 - Ocorrência de micro-organismos isolados em uroculturas coletadas em pacientes internados na Clínica Cirúrgica de um Hospital Universitário, no período de janeiro a dezembro de 2018.

Observa-se na Tabela 1, o perfil de resistência das bactérias mais frequentes, isoladas em aspirados traqueias de pacientes internados na Clínica Cirúrgica de um Hospital Universitário.

Os isolados de Pseudomonas aeruginosa apresentaram os percentuais de resistência de $22,2 \%$ para amicacina, $56 \%$ para cefepime, ceftazidina, meropenem e imipenem, $44 \%$ para ciprofloxacino e piperacilina + tazobactan e $50 \%$ levofloxacino. Porém foram sensíveis $100 \%$ a colistina (Tabela 1 ).

Ainda na (Tabela 1) pode-se observar que os isolados de Acinetobacter baumannii apresentaram os percentuais de resistência de $100 \%$ para amicacina, imipenem e meropenem e $75 \%$ ampicilina + sulbactam, porém foi $100 \%$ sensível a colistina.

Tabela 1 - Perfil de resistência das bactérias mais frequentes isoladas de aspirados traqueais coletados em pacientes internados na Clínica Cirúrgica de um Hospital Universitário, no período de janeiro a dezembro de 2018

\begin{tabular}{|c|c|c|c|c|c|c|c|c|c|c|c|c|}
\hline \multirow[t]{3}{*}{ Antimicrobianos } & \multicolumn{12}{|c|}{ Espécies bacterianas } \\
\hline & \multicolumn{2}{|c|}{$\begin{array}{c}P . \\
\text { aeruginosa }\end{array}$} & \multicolumn{2}{|c|}{$\begin{array}{c}A . \\
\text { baumannii } \\
\end{array}$} & \multicolumn{2}{|c|}{$\begin{array}{c}K . \\
\text { pneumoniae }\end{array}$} & \multicolumn{2}{|c|}{$\begin{array}{c}P . \\
\text { mirabilis }\end{array}$} & \multicolumn{2}{|c|}{$\begin{array}{c}E . \\
\text { coli } \\
\end{array}$} & \multicolumn{2}{|c|}{$\begin{array}{c}S . \\
\text { aureus }\end{array}$} \\
\hline & $\mathbf{n}$ & $\%$ & $\mathbf{n}$ & $\%$ & $\mathbf{n}$ & $\%$ & n & $\%$ & $\mathrm{n}$ & $\%$ & $\mathbf{n}$ & $\%$ \\
\hline Ampicilina & NT & NT & NT & NT & 5 & 100 & 2 & 100 & 1 & 100 & NT & NT \\
\hline Amicacina & 2 & 22,2 & 8 & 100 & 5 & 0 & 2 & 0 & 1 & 0 & NT & NT \\
\hline Amp + sub & NT & NT & 6 & 75 & 3 & 75 & 1 & 0 & 1 & 100 & NT & NT \\
\hline Amox +clav & NT & NT & NT & NT & 1 & 0 & 1 & 100 & NT & NT & NT & NT \\
\hline Cefepime & 5 & 56 & 8 & 100 & 4 & 80 & 1 & 0 & 1 & 0 & NT & NT \\
\hline Ceftarolina & NT & NT & NT & NT & NT & NT & NT & NT & NT & NT & 1 & 100 \\
\hline Cefotaxima & NT & NT & NT & NT & NT & NT & 1 & 0 & NT & NT & NT & NT \\
\hline Ceftazidina & 5 & 56 & 8 & 100 & NT & NT & NT & NT & NT & NT & NT & NT \\
\hline Cefazolina & NT & NT & NT & NT & 4 & 80 & 1 & 100 & 1 & 0 & NT & NT \\
\hline Cefoxitina & NT & NT & NT & NT & 3 & 60 & 2 & 0 & NT & NT & NT & NT \\
\hline Cefuroxima & NT & NT & NT & NT & 1 & 100 & 2 & 0 & 1 & 0 & NT & NT \\
\hline Ceftriaxona & NT & NT & NT & NT & 4 & 80 & 1 & 100 & 1 & 0 & NT & NT \\
\hline Ciprofloxacino & 4 & 44 & 8 & 100 & 4 & 80 & 2 & 0 & 1 & 0 & NT & NT \\
\hline Clindamicina & NT & NT & NT & NT & NT & NT & NT & NT & NT & NT & 1 & 100 \\
\hline Clorafenicol & NT & NT & NT & NT & NT & NT & NT & NT & NT & NT & NT & NT \\
\hline Colistina & 9 & 0 & 8 & 0 & 1 & 25 & NT & NT & 1 & 0 & NT & NT \\
\hline
\end{tabular}




\begin{tabular}{lcccccccccccc}
\multicolumn{11}{c}{ continuação } \\
\hline Ertapenem & NT & NT & NT & NT & 3 & 60 & 1 & 100 & 1 & 0 & NT & NT \\
Eritromicina & NT & NT & NT & NT & NT & NT & NT & NT & NT & NT & 1 & 100 \\
Gentamicina & 4 & 44 & 8 & 100 & 3 & 60 & 2 & 0 & 1 & 100 & NT & NT \\
Imipenem & 5 & 56 & 8 & 100 & 5 & 0 & 1 & 0 & 1 & 0 & NT & NT \\
Levofloxacino & 4 & 50 & 8 & 100 & 4 & 80 & 1 & 0 & 1 & 0 & NT & NT \\
Linezolide & NT & NT & NT & NT & NT & NT & NT & NT & NT & NT & 1 & 0 \\
Meropenem & 5 & 56 & 8 & 100 & 2 & 40 & 1 & 100 & 1 & 0 & NT & NT \\
Minociclina & NT & NT & NT & NT & NT & NT & NT & NT & NT & NT & 1 & 0 \\
Oxacilina & NT & NT & NT & NT & NT & NT & NT & NT & NT & NT & 1 & 100 \\
Penicilina & NT & NT & NT & NT & NT & NT & NT & NT & NT & NT & 1 & 100 \\
Pipe+tazo & 4 & 44 & 8 & 100 & 4 & 80 & 1 & 0 & 1 & 0 & NT & NT \\
Rifamicina & NT & NT & NT & NT & NT & NT & NT & NT & NT & NT & 1 & 0 \\
Smt+tmp & NT & NT & 8 & 100 & 3 & 75 & 1 & 0 & 1 & 0 & 1 & 0 \\
Tigeciclina & NT & NT & NT & NT & 1 & 25 & NT & NT & 1 & 0 & 1 & 0 \\
Vancomicina & NT & NT & NT & NT & NT & NT & NT & NT & NT & NT & 1 & 0 \\
NT: não testado; n: número de isolados bacterianos; $\%:$ percentual de resistência; pipe+tazo: piperacilina + tazobactam; \\
smt+tmp:sulfametoxazol + trimetoprima; amp+sub: ampicilina + sulbactam; amox+clav: amoxicilina + clavulanato.
\end{tabular}

Com relação a Klebsiella pneumoniae, foi observado resistência de $100 \%$ para ampicilina e cefuroxima, $80 \%$ para cefepime, cefazolina, ceftriaxona, ciprofloxacino, levofloxacino e piperacilina + tazobactan, $60 \%$ ertapenem, $40 \%$ para meropenem, $25 \%$ para colistina e tigeciclina. Porém, foi $100 \%$ sensível a amicacina e a amoxicilina + clavulanato (Tabela 1).

Para Proteus mirabilis, observou-se resistência de $100 \%$ para ampicilina, amoxicilina + clavulanato, cefazolina, ceftriaxona, ertapenem, meropenem e $100 \%$ de sensibilidade para amicacina, cefepime, cefotaxima, cefoxitina, cefuroxima, ciprofloxacino, gentamicina, imipenem, levofloxacino, piperacilina + tazobactan, ampicilina + sulbactam e sulfametoxazol + trimetoprima (Tabela 1).

Com relação ao percentual de resistência da Escherichia coli, foi observado $100 \%$ de resistência para ampicilina, ampicilina + sulbactam e gentamicina. No entanto, os isolados foram $100 \%$ sensível para amicacina, cefepime, cefazolina, cefuroxima, ceftriaxona, ciprofloxacino, colistina, ertapenem, imipenem, levofloxacino, meropenem, piperacilina+tazobactan, sulfametoxazol + trimetoprima etigeciclina (Tabela 1).

O isolado de Staphylococcus aureus, foi resistente a clindamicina, eritromicina, oxacilina, penicilina e sensível a linezolide, minociclina, rifamicina, sulfametoxazol + trimetoprima, tigeciclina e vancomicina (Tabela 1 ).

Na Tabela 2, observa-se o perfil de resistência aos antimicrobianos das bactérias mais frequentes isoladas em hemoculturas de pacientes internados na Clínica Cirúrgica de um Hospital Universitário.

Os isolados de Staphylococcus coagulase negativa apresentaram 100\% de resistência para ampicilina e penicilina, $86 \%$ para clindamicina, oxacilina e sulfametoxazol + trimetoprima, $29 \%$ para rifampicina e $100 \%$ de sensibilidade para linezolide, minociclina e vancomicina (Tabela 2).

Com relação a Klebsiella pneumoniae, o percentual de resistência foi de $100 \%$ para ampicilina, $50 \%$ para ampicilina + sulbactam, cefepime, cefazolina, ceftriaxona, ciprofloxacino, gentamicina, levofloxacino, piperacilina + tazobactan e sulfametoxazol + trimetoprima, $17 \%$ para cefoxitina, ertapenem, imipenem e meropenem. Entretanto, os isolados foram $100 \%$ sensíveis a amicacina, colistina e tigeciclina (Tabela 2).

Para Enterococcus faecalis observou-se $100 \%$ de resistência a ceftarolina e $100 \%$ de sensíbilidade a ampicilina, daptomicina, linezolide, penicilina, tigeciclina e vancomicina. Staphylococcus aureus apresentou 100\% de resistência para ampicilina, 50\% para clindamicina e eritromicina. Porém, foi $100 \%$ sensível a ceftarolina, daptomicina, linezolide, minociclina, oxacilina, sulfametoxazol + trimetoprima, rifampicina, 
penicilina, vancomicina e tigeciclina (Tabela 2).

A Klebsiella oxytoca demonstrou $100 \%$ de resistência para ampicilina, cefepime, amoxicilina + clavulanato, cefazolina, cefoxitina, cefuroxima, ceftriaxona, ciprofloxacino, ertapenem, gentamicina, levofloxacino, meropenem, piperacilina + tazobactane sulfametoxazol + trimetoprima. Todavia, foi $100 \%$ sensível a amicacina, doxiciclina e imipenem (Tabela 2).

Tabela 2 - Perfil de resistência das bactérias mais freqüentes isoladas de hemoculturas coletadas em pacientes internados na Clínica Cirúrgica de um Hospital Universitário, no período de janeiro a dezembro de 2018

\begin{tabular}{|c|c|c|c|c|c|c|c|c|c|c|}
\hline \multirow[t]{3}{*}{ Antimicrobianos } & \multicolumn{8}{|c|}{ Espécies bacterianas } & & \\
\hline & \multicolumn{2}{|c|}{$\begin{array}{c}\text { Staphylococcus } \\
\text { coagulase } \\
\text { negativa }\end{array}$} & \multicolumn{2}{|c|}{$\begin{array}{c}\text { K. } \\
\text { pneumoniae }\end{array}$} & \multicolumn{2}{|c|}{$\begin{array}{c}E . \\
\text { faecalis }\end{array}$} & \multicolumn{2}{|c|}{$\begin{array}{c}S . \\
\text { aureus }\end{array}$} & \multicolumn{2}{|c|}{ K. oxytoca } \\
\hline & $\mathbf{n}$ & $\%$ & $\mathbf{n}$ & $\%$ & $\mathbf{n}$ & $\%$ & $\mathbf{n}$ & $\%$ & $\mathbf{n}$ & $\%$ \\
\hline Ampicilina & 2 & 100 & 6 & 100 & 2 & 0 & 1 & 100 & 1 & 100 \\
\hline Amicacina & NT & NT & 6 & 0 & NT & NT & NT & NT & 1 & 0 \\
\hline Amp+sub & NT & NT & 3 & 50 & NT & NT & NT & NT & NT & NT \\
\hline Amox +clav & NT & NT & NT & NT & NT & NT & NT & NT & 1 & 100 \\
\hline Cefepime & NT & NT & 3 & 50 & NT & NT & NT & NT & 1 & 100 \\
\hline Ceftazidina & NT & NT & NT & NT & NT & NT & NT & NT & NT & NT \\
\hline Cefazolina & NT & NT & 3 & 50 & NT & NT & NT & NT & 1 & 100 \\
\hline Cefoxitina & NT & NT & 1 & 17 & NT & NT & NT & NT & 1 & 100 \\
\hline Cefuroxima & NT & NT & NT & NT & NT & NT & NT & NT & 1 & 100 \\
\hline Ceftriaxona & NT & NT & 3 & 50 & NT & NT & NT & NT & 1 & 100 \\
\hline Ceftarolina & NT & NT & NT & NT & 1 & 100 & 2 & 0 & NT & NT \\
\hline Ciprofloxacino & NT & NT & 3 & 50 & NT & NT & NT & NT & 1 & 100 \\
\hline Clindamicina & 6 & 86 & NT & NT & NT & NT & 1 & 50 & NT & NT \\
\hline Colistina & NT & NT & 6 & 0 & NT & NT & NT & NT & NT & NT \\
\hline Doxiciclina & NT & NT & NT & NT & NT & NT & NT & NT & 1 & 0 \\
\hline Daptomicina & NT & NT & NT & NT & 2 & 0 & 2 & 0 & NT & NT \\
\hline Ertapenem & 6 & 86 & 1 & 17 & NT & NT & NT & NT & 1 & 100 \\
\hline Eritromicina & NT & NT & NT & NT & NT & NT & 1 & 50 & NT & NT \\
\hline Gentamicina & NT & NT & 3 & 50 & NT & NT & NT & NT & 1 & 100 \\
\hline Imipenem & NT & NT & 1 & 17 & NT & NT & NT & NT & 1 & 0 \\
\hline Levofloxacino & NT & NT & 3 & 50 & NT & NT & NT & NT & 1 & 100 \\
\hline Linezolide & 7 & 0 & NT & NT & 2 & 0 & 2 & 0 & NT & NT \\
\hline Meropenem & NT & NT & 1 & 17 & NT & NT & NT & NT & 1 & 100 \\
\hline Minociclina & 7 & 0 & NT & NT & NT & NT & 2 & 0 & NT & NT \\
\hline Oxacilina & 6 & 86 & NT & NT & NT & NT & 2 & 0 & NT & NT \\
\hline Pipe+tazo & NT & NT & 3 & 50 & NT & NT & NT & NT & 1 & 100 \\
\hline Penicilina & 2 & 100 & NT & NT & 2 & 0 & 1 & 0 & NT & NT \\
\hline Rifampicina & 2 & 29 & NT & NT & NT & NT & 2 & 0 & NT & NT \\
\hline $\mathrm{Smt}+\mathrm{tmp}$ & 6 & 86 & 3 & 50 & NT & NT & 2 & 0 & 1 & 100 \\
\hline Tigeciclina & NT & NT & 6 & 0 & 2 & 0 & 2 & 0 & NT & NT \\
\hline Vancomicina & 7 & 0 & NT & NT & 2 & 0 & 2 & 0 & NT & NT \\
\hline
\end{tabular}

NT: não testado; n: número de isolados bacterianos; \%: percentual de resistência; pipe+tazo: piperacilina + tazobactam; smt+tmp:sulfametoxazol + trimetoprima; amp+sub: ampicilina + sulbactam; amox+clav: amoxicilina + clavulanato.

Na Tabela 3, pode-se observar o perfil de resistência aos antimicrobianos das bactérias mais frequentes isoladas nas uroculturas de pacientes internados na Clínica Cirúrgica de um Hospital Universitário.

A Escherichia coli apresentou um percentual de $100 \%$ de resistência para ciprofloxacino, nitrofurantoina, norfloxacino e tetraciclina, $67 \%$ para ampicilina e cefotaxima, $62 \%$ para levofloxacino, 58\% para ampicilina + sulbactam, 56\% para cefuroxima, $40 \%$ para ceftriaxona, $38 \%$ para sulfametoxazol + trimetoprima, $33 \%$ para amoxicilina + clavulanato e cefepime, $28 \%$ para gentamicina, $11 \%$ para fosfomicina g6p e $7 \%$ para meropenem. Todavia, esses isolados apresentaram $100 \%$ de sensibilidade ao aztreonam, amicacina, cefoxitina, colistina, ertapenem, imipenem, piperacilina + tazobactan e tigeciclina (Tabela 3).

Klebsiella pneumoniae, demonstrou $100 \%$ de resistência para ampicilina, cefalotina, cefotaxima, norfloxacino, $73 \%$ para cefepime, $69 \%$ para ampicilina + sulbactam, $50 \%$ para nitrofurantoina, $47 \%$ para levofloxacino, $40 \%$ para piperacilina+tazobactan, 
sulfametoxazol + trimetoprima, $38 \%$ para gentamicina e cefoxitina, $27 \%$ para meropenem e ertapenem; $19 \%$ para imipenem, $17 \%$ para colistina e tigeciclina, mas apresentou sensibilidade de $100 \%$ para amicacina (Tabela 3 ).

Pseudomonas aeruginosa, demonstrou $100 \%$ de resistência para ceftazidina, doxiciclina e sulfametoxazol + trimetoprima; $88 \%$ para cefepime, $86 \%$ paraimipenem, $75 \%$ para meropenem e ciprofloxacino, 63\% para gentamicina, $20 \%$ para colistina, $14 \%$ para levofloxacino e 13\% para piperacilina + tazobactan. Mas apresentou $100 \%$ de sensibilidade ao norfloxacino (Tabela 3 ).

Em relação aos isolados de Acinetobacter baumannii, foi observado $100 \%$ de resistência para ampicilina, cefepime, ceftazidina, ciprofloxacino, gentamicina, imipenem, levofloxacino, meropenem e piperacilina + tazobactan, $50 \%$ para amicacina, cefazolina e sulfametoxazol + trimetoprima (Tabela 3 ).

Enterococcus faecium, demosntrou 50\% de resistência para ampicilina, ciprofloxacino, piperacilina + tazobactan, mas foi sensível 100\% sensível a daptomicina, linezolide, nitrofurantoina e vancomicina (Tabela 3 ).

Tabela 3 - Perfil de resistência das bactérias mais frequentes isoladas de uroculturas coletadas em pacientes internados na Clínica Cirúrgica de um Hospital Universitário, no período de janeiro a dezembro de 2018

\begin{tabular}{|c|c|c|c|c|c|c|c|c|c|c|}
\hline \multirow[t]{3}{*}{ Antimicrobianos } & \multicolumn{6}{|c|}{ Espécies bacterianas } & & & & \\
\hline & \multicolumn{2}{|c|}{$\begin{array}{c}E . \\
\text { coli }\end{array}$} & \multicolumn{2}{|c|}{$\begin{array}{c}K . \\
\text { pneumoniae }\end{array}$} & \multicolumn{2}{|c|}{$\begin{array}{c}P . \\
\text { aeruginosa }\end{array}$} & \multicolumn{2}{|c|}{ A. baumannii } & \multicolumn{2}{|c|}{$\begin{array}{c}E . \\
\text { faecium }\end{array}$} \\
\hline & $\mathbf{n}$ & $\%$ & n & $\%$ & $\mathbf{n}$ & $\%$ & n & $\%$ & n & $\%$ \\
\hline Ampicilina & 12 & 67 & 16 & 100 & NT & NT & 2 & 100 & 1 & 50 \\
\hline Amicacina & 18 & 0 & 16 & 0 & 4 & 50 & 1 & 50 & NT & NT \\
\hline Aztreonam & 1 & 0 & NT & NT & NT & NT & NT & NT & NT & NT \\
\hline Amp+sub & 7 & 58 & 9 & 69 & NT & NT & NT & NT & NT & NT \\
\hline Amox +clav & 2 & 33 & 3 & 100 & NT & NT & NT & NT & NT & NT \\
\hline Cefalotina & 3 & 100 & 1 & 100 & NT & NT & NT & NT & NT & NT \\
\hline Cefepime & 5 & 33 & 11 & 73 & 7 & 88 & 2 & 100 & NT & NT \\
\hline Cefotaxima & 2 & 67 & 1 & 100 & NT & NT & NT & NT & NT & NT \\
\hline Ceftazidina & NT & NT & NT & NT & 7 & 100 & 2 & 100 & NT & NT \\
\hline Cefazolina & 5 & 100 & 9 & 100 & NT & NT & 1 & 50 & NT & NT \\
\hline Cefoxitina & 16 & 0 & 6 & 38 & NT & NT & NT & NT & NT & NT \\
\hline Cefuroxima & 5 & 56 & 4 & 100 & NT & NT & NT & NT & NT & NT \\
\hline Ceftriaxona & 6 & 40 & 11 & 73 & NT & NT & NT & NT & NT & NT \\
\hline Ciprofloxacino & 1 & 100 & 8 & 100 & 6 & 75 & 2 & 100 & 1 & 50 \\
\hline Colistina & 7 & 0 & 2 & 17 & 1 & 20 & NT & NT & NT & NT \\
\hline Doxiciclina & NT & NT & NT & NT & 2 & 100 & NT & NT & NT & NT \\
\hline Daptomicina & NT & NT & NT & NT & NT & NT & NT & NT & 2 & 0 \\
\hline Ertapenem & 14 & 0 & 4 & 27 & NT & NT & NT & NT & NT & NT \\
\hline Fosfomicina $\mathrm{g} 6 \mathrm{p}$ & 1 & 11 & NT & NT & NT & NT & NT & NT & NT & NT \\
\hline Gentamicina & 5 & 28 & 6 & 38 & 5 & 63 & 2 & 100 & NT & NT \\
\hline Imipinem & 17 & 0 & 3 & 19 & 6 & 86 & 2 & 100 & NT & NT \\
\hline Levofloxacino & 8 & 62 & 7 & 47 & 1 & 14 & 2 & 100 & NT & NT \\
\hline Linezolide & NT & NT & NT & NT & NT & NT & NT & NT & 2 & 0 \\
\hline Meropenem & 1 & 7 & 4 & 27 & 6 & 75 & 2 & 100 & NT & NT \\
\hline Nitrofurantoina & 4 & 100 & 2 & 50 & NT & NT & NT & NT & 1 & 0 \\
\hline Norfloxacino & 4 & 100 & 1 & 100 & 1 & 0 & NT & NT & NT & NT \\
\hline Pipe+tazo & 15 & 0 & 6 & 40 & 1 & 13 & 2 & 100 & NT & NT \\
\hline Penicilina & NT & NT & NT & NT & NT & NT & NT & NT & 1 & 50 \\
\hline Smt+tmp & 6 & 38 & 6 & 40 & 1 & 100 & 1 & 50 & NT & NT \\
\hline Tigeciclina & 7 & 0 & 2 & 17 & NT & NT & NT & NT & NT & NT \\
\hline Tetraciclina & 1 & 100 & NT & NT & NT & NT & NT & NT & NT & NT \\
\hline Vancomicina & NT & NT & NT & NT & NT & NT & NT & NT & 2 & 0 \\
\hline
\end{tabular}

NT: não testado; n: número de isolados bacterianos; \%: percentual de resistência; pipe+tazo: piperacilina + tazobactam; smt+tmp:sulfametoxazol + trimetoprima; amp+sub: ampicilina + sulbactam; amox+clav: amoxicilina + clavulanato.

\section{Discussão}

Em relação a incidência de bactérias em aspirados traqueais, um estudo realizado por Pires et al. 2010 (13) na enfermaria do Hospital das Clínicas (HC) da Universidade 
Federal de Pernambuco observou que os micro-organismos com maior incidência nessas amostras foram $P$. aeruginosa, $S$. aureus e em menor quantidade foram $K$. pneumoniae, $P$. mirabilis e E. coli, corroborando com achados do presente trabalho. Nesse mesmo estudo, a análise do perfil de sensibilidade aos antimicrobianos demonstrou que a $P$. aeruginosa apresentou, em geral, boa sensibilidade aos antimicrobianos, porém foram resistentes ao imipenem $(81,8 \%)$ e ao meropenem $(79,3 \%)$. No presente estudo foi observado um menor percentual de resistência para imipenem e meropenem de $56 \%$.

Acredita-se que a resistência observada nas espécies de P. aeruginosa, seja devido aos diferentes mecanismos, dentre eles, pode-se citar, a resistência aos $\beta$-lactâmicos, que se deve a produção de enzimas $\beta$-lactamases, hiperexpressão de sistemas de efluxo, alteração da permeabilidade da membrana e pela síntese de proteínas de ligação à penicilina (PBPs) com baixa afinidade por $\beta$-lactâmicos (14). Sendo importante frisar que essa espécie, pode apresentar todos esses mecanismos e que podem existir simultaneamente, daí sua importância clínica (15).

Vale ressaltar, que os dados do presente estudo são referentes amostras colhidas na Clínica Cirúrgica e grande parte dos estudos refere-se somente aqueles internados na UTI. Todavia, assim como o trabalho citado acima, no presente estudo, a bactéria mais frequente nos aspirados traqueais foi a $P$. aeruginosa, podendo ser explicado devido a presença de pacientes traqueostomizados neste ambiente. Sabe-se que $P$. aeruginosa está associada ao uso de ventiladores mecânicos (16). Outras características relevantes desta bactéria é o fato de ser altamente versátil, capaz de tolerar condições de baixo oxigênio, sobreviver com baixos níveis de nutrientes e crescer em faixas de temperatura de 4 a $42^{\circ} \mathrm{C}$. Ainda possui mecanismos, os quais permitem que ela fique aderida e sobreviva em equipamentos médicos e outras superfícies hospitalares(17).

Em relação aos isolados de $A$. baumannii, pesquisadores relataram que o sítio de infecção com maior prevalência (35\%) foi em aspirados traqueais, em comparação a hemoculturas e uroculturas (18). Esses dados corroboram com o presente estudo, onde foi obtido um percentual de $25,8 \%$ nos aspirados e $1,3 \%$ em uroculturas. Com relação ao perfil de resistência aos antibióticos, Ricas et al. 2014 (18), observaram, um percentual de cefepime (81\%), ceftazidima $(83,7 \%)$, ceftriaxona $(82,6 \%)$, amicacina $(67,4 \%)$, imipenem $(72,3 \%)$ e para meropenem $(75,5 \%)$. Neste estudo foi encontrado um percentual de $100 \%$ de resistência para esses mesmos medicamentos.

Esse percentual de resistência para pacientes internado na Clínica Cirúrgica do Hospital Universitário chama atenção, uma vez que, era de se esperar resultados inferiores, devido a uma menor incidência de infecção hospitalar, bem como, uma menor utilização de antibióticos, o que acarretaria um percentual menor de resistência bacteriana.

A importância dessa bactéria tem sido discutida nos últimos anos em decorrência da sua grande capacidade em adquirir mecanismos de resistência às diferentes classes de antibióticos, além de apresentar características, como, aptidão em sobreviver e se adaptar a condições adversas. Sendo assim, a união desses fatores torna essa bactéria responsável por uma morbidade e mortalidade elevada, nos pacientes críticos (19). Essa resistência aos carbapenêmico pode ser consequência da combinação de diferentes mecanismos, que são: diminuição da permeabilidade das membranas externas, alteração na afinidade das proteínas ligadoras de penicilinas (PBPs) e, raramente, pela hiper-expressão de bombas de efluxo (20). Além destes micro-organismos pode ocorrer a expressão de metalo- $\beta$ lactamases (MBLs), as quais não são hidrolisadas por inibidores comerciais e a expressão de oxacilinases (21).

Vale ressaltar, que a disseminação de espécies multirresistentes colocou em risco a utilização de diferentes fármacos, sendo então utilizado como alternativa, a ampicilina + sulbactam como uma opção terapêutica para o tratamento de cepas carbapenêmicos- 
resistentes (22).

A bactéria $P$. mirabilis, aparece de forma discreta $(6,4 \%)$ nos aspirados traqueais do presente estudo, mas ainda com uma ocorrência maior, quando comparado com os dados de Souza, 2011 (23) com 0,9 \% e de Debarba et al. 2018 (24) com $2 \%$.

Siqueira et al. 2011 (25), observaram que nas amostras de hemoculturas, o agente patogênico mais encontrado, foi o Staphylococcus coagulase negativa, corroborando com os dados do presente trabalho. Sabe-se que esse grupo de bactérias pode ser considerado contaminante em hemocultura, devido colonizarem a pele, o que justifica a sua elevada ocorrência nas amostras de hemocultura (26).

Com relação ao seu perfil de resistência, Perez e D'azevedo 2008 (27) afirmam que 70\% a $90 \%$ dos isolados de SCN apresentaram resistência à oxacilina. No presente trabalho foi observado um percentual de $86 \%$ para este antibiótico. Ainda de acordo com os dados de Rigatti et al. 2010 (26), os SCN foram 100\% sensível à vancomicina, igual os dados deste trabalho. A sensibilidade para este antibiótico é relevante, uma vez que, devido ao surgimento de cepas de Enterococcus sp. resistentes à vancomicina (VRE) e Staphylococcus sp. com sensibilidade reduzida à vancomicina, a redução do uso deste antimicrobiano têm sido recomendadas. Sendo assim, os fármacos como, linezolide, telavancina, oritavancin e dalbavancin surgem como novas opções terapêuticas permitindo o uso resguardado da vancomicina (26).

De acordo com Leão et al. 2007 (28), foi encontrado entre as 44 hemoculturas analisadas, um percentual de $13,6 \%$ para $S$. aureus e 6,8\% para E. faecalis nas amostras de pacientes críticos. Com relação ao percentual de resistência, todos os isolados de $S$. aureus foram resistentes a amplicilina, além de $66,7 \%$ para a oxacilina, corroborando em parte com os dados do presente trabalho, já que nossos isolados foram sensíveis a oxacilina. Neste mesmo estudo, todas as bactérias gram positivas, incluído as duas bactérias citadas acima, foram sensíveis ao linezolida e à vancomicina, corroborando com os dados do presente trabalho. Essa sensibilidade observada é importante, já que o surgimento de cepas de $S$. aureus resistentes à vancomicina (VERSA) foram recentemente descobertas. Para Chang et al. 2003 (29) essa resistência teria sido adquirida por meio da transferência de um plasmídeo contendo o transposon do gene vanA, Tn1546, de um E. faecalis resistente à vancomicina para essa bactéria já resistente à oxacilina (MRSA), que já possuía um plasmídeo codificando resistência à gentamicina e produção de $\beta$-lactamase (30). Ao longo do tempo, novas cepas com essas características foram descobertas, sendo desta forma, recomendado o uso de linezolida em substituição à vancomicina. Vale ressaltar, que apesar dessa nova opção de antibiótico, é vital evitar o uso de vancomicina de forma abusiva e desnecessária, além de adotar medidas de vigilância epidemiológica, sobre os serviços de saúde (31).

Em um estudo realizado por Bortoli 2011 (32) na enfermaria geriátrica do hospital do servidor público municipal em São Paulo, foi observado que as uroculturas dos pacientes apresentaram como principal micro-organismo a E. coli com uma incidência de $30 \%$ (46 amostras). Corroborando com os dados deste estudo, onde foi observado uma ocorrência de 28,2\%. Conforme De Santana et al. 2017 (33) foi observado que E. coli foi à bactéria mais frequentemente isolada $(44,4 \%)$ tanto entre as infecções de origem ambulatorial quanto nas de pacientes hospitalizados. Neste mesmo sentido, De Freitas 2016 (34) observou que dentre os pacientes atendidos no Instituto Lauro de Souza Lima, Bauru SP, o agente patogênico mais encontrado em uroculturas foi a $E$. coli presente em $54,8 \%$ dos casos. Ambas as unidades de serviço, tanto ambulatorial quanto hospitalar, apresentaram essa bactéria como agente etiológico predominante, porém uma maior incidência foi verificada para as infecções de origem comunitária, com 67,1\% contra apenas $39,1 \%$ nas enfermarias. Este estudo verificou que a bactéria $E$. coli apresentou 
altas taxas de resistência à penicilina $\mathrm{G}(95,3 \%)$, tetraciclina $(60 \%)$, cefalotina $(61,9 \%)$, sulfametoxazol + trimetoprim (50\%), amoxicilina + ácido clavulânico $(48,5 \%)$ e às quinolonas (ácido nalidixílico, norfloxacina, ciprofloxacino, ofloxacina e levofloxacina) com taxas entre $40 \%$ a $60 \%$ de resistência. No presente estudo, foi encontrada uma maior resistência para tetraciclina, cefalotina, ciprofloxacino e levofloxacina. A elevada resistência à cefalotina é relevante, pois tal antibiótico está entre os fármacos de primeira escolha para o tratamento de cistite em gestantes nas quais o uso de antibióticos deve ser limitado (35). A resistência ao sulfametoxazol + trimetoprim tem aumentado em todo o mundo, mesmo nas infecções de origem comunitária, o que vem limitando seu uso como antibiótico empírico $(36,37)$. Apesar dessa informação, no presente estudo a resistência foi de $38 \%$ apenas.

A resistência de $E$. coli, aos antibióticos $\beta$-lactâmicos na década de 90 estava relacionada essencialmente pela produção de $\beta$-lactamases de espectro estendido (ESBLs) do tipo TEM ou SHV, também conhecidas como ceftazidimases, codificadas por plasmídeos. Estas enzimas possuem a capacidade de hidrolisar penicilinas e cefalosporinas, com maior atividade hidrolítica para a ceftazidima (entre as cefalosporinas de largo espectro) e possui a capacidade de ser inibidas pelo ácido clavulânico. Mais tarde há o surgimento de um novo grupo de enzimas, o CTX-M que se tornou mais frequente em países da Europa e América do Sul (38). Além da resistência, fatores intrínsecos contribuem para a complexa interação entre o hospedeiro e o agente patogênico nas infecções urinárias. Fatores de virulência expressos por este micro-organismo o torna responsável por infecções urinarias severas (39).

Ainda em relação às uroculturas, Da Silva et al. (40) em seu estudo observaram uma maior prevalência de $P$. aeruginosa (32,5\%). Em pacientes internados na UTI, essa bactéria é considerada responsável por infecções oportunistas. Nesse mesmo trabalho foram observadas para essa espécie bacteriana, percentuais de resistência de $96 \%$ para ciprofloxacino, $93 \%$ para trobamicina, $92 \%$ para levofloxacina e $88 \%$ para ceftriaxona. No presente estudo observou-se uma resistência inferior para ciprofloxacino (75\%) e levoflaxacina $(14 \%)$

Esta bactéria é considerada a principal causa de infecções hospitalares dentre os bacilos gram-negativos não fermentadores de glicose. Por apresentar diferentes mecanismos de resistência ela causa um impacto clínico considerável já que leva ao comprometimento da eficácia de quase todas as drogas utilizadas no tratamento contra ela (41).

Barros et al. 2013 (42), encontraram entre as bactérias mais frequentes isoladas nas uroculturas o A. baumannii $(9,7 \%)$, nesse mesmo estudo, também foi encontrado um percentual de $83,3 \%$ de resistência para o imipenem e meropenem. No presente estudo o percentual de resistência para ambos os fármacos foi de $100 \%$.

Em nosso estudo, foi observado a presença $K$. pneumoniae entre as 3 bactérias mais incidentes nos isolados bacterianos dos aspirados traqueais (16,1\%), hemocultura $(22,2 \%)$ e uroculturas $(25 \%)$. Este patógeno é caracterizado por ser um bacilo gramnegativo e sua resistência a antimicrobianos é considerado um problema de saúde pública e vem causando preocupação em todos os campos da saúde (43).

A sua resistência pode ser originada de diversas formas, dentre elas, o uso inadequado de antimicrobianos e a produção de $\beta$-lactamases tipo AmpC e de carbapenemases, como as metalo- $\beta$-lactamases (MBL) e carbapenemasesdo tipo KPC. Grande parte das infecções associadas à $K$. pneumoniae, produtora da enzima KPC, ocorre em pacientes imunodeprimidos hospitalizados e/ou com dispositivos invasivos, sendo assim, a prevenção é a principal arma usada no combate desta bactéria (44).

De maneira geral, os dados do presente estudo corroboram com grande maioria dos perfis de resistência das bactérias de diversos hospitais espalhados pelo Brasil. Foi 
observado que na Clínica Cirúrgica do Hospital Universitário há diferentes espécies bacterianas que causam infecções hospitalares e que a grande maioria apresenta uma elevada resistência aos antibióticos.

\section{Conclusão}

Os dados permitem o conhecimento do perfil bacteriano das amostras de aspirados traqueais, hemoculturas e uroculturas dos pacientes internados na Clínica Cirúrgica, o que poderá nortear o tratamento empírico das infecções e consequentemente diminuir a seleção de bactérias multirresistentes, auxiliando na prevenção e no controle das infecções hospitalares.

\section{Referências}

1. Horan TC, Andrus M, Dudeck M. A. Surveillance definition of healthcare-associated infection and criteria for specific types of infections in the acute care setting. American Journal of Infection Control 2008; 36: 311-333.

2. Oliveira AC, Paula AO, Iquiapaza, RA, Lacerda, ACS. Infecções relacionadas à assistência em saúde e gravidade clínica em uma unidade de terapia intensiva. Revista Gaúcha de Enfermagem 2012; 33: 89-96.

3. Rutalla AW, White MS, Gergen MF, Weber DJ. Bacterial contamination of keyboards: efficacy and functional impact of disinfectants. Infection Control \& Hospital Epidemiology 2006; 27: 372-377.

4. Menegueti MG, Canini SEMS, Rodrigues FB, Laus AM. Avaliação dos Programas de Controle de Infecção Hospitalar em serviços de saúde. Rev. Latino-Am. Enfermagem 2015; 23(1): 98-105.

5. Turrini RNT. Percepção das Enfermeiras sobre fatores de risco para a infecção hospitalar. Rev Esc Enf. USP 2000; 34(2): 174-84.

6. Allegranzi B, Nejad SB, Combescure C, Graafmans W, Attar H, Donaldso L, et al. Burden of endemic health-care-associated infection in developing countries: systematic review and meta-analysis. The Lancet 2011; 377(9761): 228-41.

7. Estrela T S. Resistência antimicrobiana: enfoque multilateral e resposta brasileira. Saúde e Política Externa: os 20 anos da Assessoria de Assuntos Internacionais de Saúde (1998 - 2018). Rio de janeiro. 2018.

8. Rosa MB, Reis AMM, Lima CR. A Farmácia e o Controle das Infecções Hospitalares. In: GOMES, M J V M. \& REIS, A M M. (Org). Ciências Farmacêuticas: Uma Abordagem em Farmácia Hospitalar. São Paulo: Atheneu; 2003; p.407-428.

9. Quian Q, Venkataraman L, Kirby JE, Gold HS, Yamazumi T. Direct Detection of Methicillin Resistance in Staphylococcus aureus in Blood Culture Broth by Use of a Penicillin Binding Protein 2a Latex Agglutination Test. Journal of Clinical Microbiology 2010; 48(140): 1420-1421.

10. Hoecke FV, Deloof NE, Claeys G. Performance evaluation of a modified chromogenic medium, ChromID MRSA New, for the detection of methicillin-resistant Staphylococcus aureus from clinical specimens. European Journal of Clinical Microbiology and Infectious Diseases 2011; 30(12) 15951598.

11. Queenan AM, BushK. Carbapenemases: the Versatile B-Lactamases. Clinical Microbiology 2007; 20:440-458.

12. Peirano G, Seki LM, Passos VLV, Pinto MCFG, Guerra LR, Asensi MD. Carbapenem-hydrolysing beta-lactamase KPC-2 in Klebsiella pneumoniae isolated in Rio de Janeiro, Brazil. Journal of Antimicrobial Chemotherapy 2009; 63: 265-268.

13. Pires EJVC, Júnior VVDS, Lopes ACDS, Veras DL, Leite LE, Maciel, MAV. Análise epidemiológica de isolados clínicos de Pseudomonas aeruginosa provenientes de hospital universitário. Revista Brasileira de Terapia Intensiva 2010; 21: 384-390.

14. Fuentefria DB. Detecção de metalo $\beta$-lactamases e similaridadegenética em isolados de Pseudomonasaeruginosa de efluentehospitalar e água superficial. porto alegre. tese [Doutorado em 
Microbiologia Agrícola e do Ambiente] - Instituto de Ciências Básicas da Saúde da Universidade Federal do Rio Grande do Sul; 2009.

15. Strateva T, Yordanov D. Pseudomonas aeruginosa- a phenomenon of bacterial resistance. J Med Microbiol 200958 (9):1133-48.

16. Bush LM. [Internet]. Infecções por Pseudomonas.2019[citado em dezembro2019] disponível em: http://www.msdmanuals.com/ptbr/profissional/doençasinfecciosas/bacilos-gramnegativos/pseudomonas-e-infecções-relacionadas.

17. Ochoa, AS, López-Montiel, F, Escalona G, Cruz-córdova A, Dávila LB, López-Martínez B. Pathogenic characteristics of Pseudomonas aeruginosa strains resistant to carbapenems associated with biofilm formation. Boletin medico del hospital infantil de Mexico 2013; 70(2): 138-150.

18. Ricas RV, Marques TC, Yamamoto ACA. Perfil de resistência de Acinetobacter baumannii a antimicrobianos em um hospital universitário de Cuiabá-MT. Infarma Ciências Farmacêuticas 2013; 25(4): 178-81.

19. Falagas ME, Bliziotis IA, Siempos II. Attributable mortality of Acinetobacter baumannii infections in critically illpatients: a systematic review of matched cohort and case-control studies. CritCare 2006:10(2);48.

20. Gusatti CDS, Ferreira AE, Fuentefria BD, Corção G. Resistência a $\beta$-lactâmicos em Acinetobacter spp isolados de efluente hospitalar no sul do brasil. Revista da Sociedade Brasileira de Medicina Tropical 2009;42(2): 183-187.

21. Walsh TR, Toleman MA, Poirel L, Nordmann P. Metallo- $\beta$-lactamases: the quiet before the storm. Clinical Microbiology Reviews 2005; 18:306-325.

22. Viana GF, Saalfeld SM, Moreira RR, Menegucci TC, Garcia LB, Cardoso CL, et al. Canampicillin/sulbactram resistance in Acinetobacter baumannii be predicted accurately by disk diffusion? J. Glob Antimicrob Resist. 2013;1(4):221-22.

23. Souza GM. [Internet] Prevalência demicrorganismo em secreção traqueal em pacientes da UTI de hospital público. 2011. [Acessado em novembro 2019] disponível em: https://www.webartigos.com/artigos/prevalencia-de-microrganismos-em-secrecao-traqueal-empacientes-da-uti-de-hospital-publico/80302.

24. Debarba E, Silvero KSV, Teixeira JJV, da Silva CM, de Peder, LD. Prevalência microbiana em secreções traqueais de pacientes em unidade de terapia intensiva-experiência de 4 anos. Journal of Infection Control 2018;(7)1: 1-14.

25. Siqueira GLG, Hueb W, Contreira R, Nogueron, MA, Cancio, DM,Caffaro RA Infecção de corrente sanguínea relacionada a cateter venoso central (ICSRC) em enfermarias: estudo prospectivo comparativo entre veia subclávia e veia jugular interna. Jornal Vascular Brasileiro 2011;10(3): 211-216.

26. Rigatti, F, Tizotti, MK, Hörner R, Domingues VO, Martini R, Mayer LE, Da costa MM. Bacteremias por Staphylococcus coagulase negativos oxacilina resistentes em um hospital escola na cidade de santa maria, estado do rio grande do sul. Revista da Sociedade Brasileira de Medicina Tropical 2010;43(6): 686-690.

27. Perez LRR, D'azevedo, PA. Evaluation of the accuracy of various phenotypic tests to detect oxacillinresistance in coagulase-negative Staphylococci. Brazilian Journal of Infectious Diseases 2008; 12(3): 210-212.

28. Leão LSNDO, Passos XS, Reis C, Valadão LMA, Silva MDRR, Pimenta, FC. Fenotipagem de bactérias isoladas em hemoculturas de pacientes críticos. Revista da Sociedade Brasileira de Medicina Tropical 2007;40(5): 37-540.

29. Chang S, Sievert DM, Hageman JC, Boulton ML, Tenover FC, et al. Infection with vancomycin resistant Staphylococcus aureus containing the van A resistance gene. N Engl J Med 2003; 348: 13427.

30. Weigel LM, Clewell DB, Gill SR, Clark NC, Mcdouga LK, Flannaganse SE, et al. Genetic analysis of a high-level vancomycin-resistant isolate of Staphylococcus aureus. Science 2003; 302:1569-71.

31. Mimica MJ, Berezin EM. Staphylococcus aureus resistente à vancomicina: um problema emergente. Arquivos médicos dos hospitais e da Faculdade de Ciências Médicas da Santa Casa de São Paulo 2018; 51(2): 52-56.

32. Bortoli, AAMD. Perfil de sensibilidade bacteriana em uroculturas de pacientes internados na enfermaria geriátrica do hospital do servidor público municipal. 2011. 24p.tese (residência médica). 
São Paulo.

33. De Santana TCFS, Pereira EMM, Monteiro SG, Carmo MS, Turri RJG, Figueiredo PMS. Prevalência e resistência bacteriana aos agentes antimicrobianos de primeira escolha nas infecções do trato urinário no município de São Luís-MA. Revista de Patologia Tropical 2017; 41:44.

34. De Freitas BVL, Germino RV, Trino, LM, Diório SM, Fusar AE. Prevalência e perfil de susceptibilidade a antimicrobianos de uropatógenos em pacientes atendidos no instituto Lauro de Souza lima, Bauru, sp. Revista Brasileira de Análises Clínicas 2016;48:375-380.

35. Ovalle A, Levancini M. Urinary tract infections in pregnancy. Current opinion in urology 2008; 11: 55-59.

36. Moreira MAA, Costa FS, Nogueira NAP. Bacteriúria assintomática em gestantes atendidas no centro de saúde ambulatorial Abdornal Machado (CESA-AM) em Cratéus, CE. Revista Brasileira de Análises Clínicas 2003; 35:19-21.

37. Braoios A, Turrati TF, Meredija LCS, Campos TRS, Denadai FHM. Infecções do trato urinário em pacientes não hospitalizados: etiologia e padrão de resistência aos antimicrobianos. J Bras Patol Med Lab 2009; 45 (6): 449-456.

38. Brange RC, Zamfir O, Geoffroy S, et al. Genetic background of Escherichia coli and extendedspectrum $\beta$-lactamasetype. Emerg Infect Dis 2005; 11(1): 54-61.

39. Narciso A, Lito L, Cristino JM, Duarte A, Norte CHL, Duarte A. Escherichia coli uropatogénica: resistência aos antibióticos versus factores de virulência. Acta Urol 2010; 27(2): 11-20.

40. Da Silva FV, Kimura CA, Coimbra, MVS. Perfil de sensibilidade antimicrobiana das Pseudomonas aeruginosa isoladas de pacientes da unidade de tratamento intensiva de um hospital público de Brasília. Revista de Divulgação Científica Sena Aires 2012;1(1): 19-24.

41. Santos IAL, Nogueira JMR, Mendonça FCR. Mecanismos de resistência antimicrobiana em Pseudomonas aeruginosa. RBAC: Revista Brasileira de Análises Clínicas 2015;(47):5-12.

42. Barros SKSDA, Kerbauy G, Dessunti EM. Infecção do trato urinário relacionada ao cateter: perfil de sensibilidade antimicrobiana. Rev Rene 2013; 14(4): 1005-13.

43. Moreira VC, Freire D. [Internet] Klebsiella pneumoniaee sua resistência a antibióticos. [ Acessado em novembro 2019 ] disponível em: http://www. cpgls. ucg. br/6mostra/artigos/saude. vaness a\% 20carvalho\% 20moreira. pdf.

44. Marchaim D, Navon-Venezia S, Schwaber MJ, Carmeli Y. Isolation of imipenem-resistant enterrobacter species; emergence of kpc-2 carbapenemase, molecular characterization, epidemiology, and outcomes. Antimicrob Agentes Chemother 2008; 52: 1413-1418. 